\title{
BSM fits for rare $B$ decays
}

\section{Farvah Mahmoudi*}

Univ Lyon, Univ Lyon 1, CNRS/IN2P3, Institut de Physique Nucléaire de Lyon UMR5822,

F-69622 Villeurbanne, France

Theoretical Physics Department, CERN, CH-1211 Geneva 23, Switzerland

E-mail: nazila@cern.ch

\author{
Alexandre Arbey \\ Univ Lyon, Univ Lyon 1, CNRS/IN2P3, Institut de Physique Nucléaire de Lyon UMR5822, \\ F-69622 Villeurbanne, France \\ Theoretical Physics Department, CERN, CH-1211 Geneva 23, Switzerland \\ E-mail: alexandre.arbeyeens-lyon.fr
}

\section{Tobias Hurth}

PRISMA Cluster of Excellence and Institute for Physics (THEP) Johannes Gutenberg University, D-55099 Mainz, Germany

E-mail: tobias.hurth@cern.ch

\section{Siavash Neshatpour}

School of Particles and Accelerators, Institute for Research in Fundamental Sciences (IPM), P.O. Box 19395-5531, Tehran, Iran

E-mail: neshatpourdipm.ir

The $\mathrm{LHCb}$ experiment has made several measurements in $b \rightarrow s$ transitions which indicate tensions with the Standard Model predictions. We examine how the significance of the tensions in $B \rightarrow K^{*} \mu^{+} \mu^{-}$observables depend on the various theory implementations of the long-distance effects. We also consider a general parameterisation, consistent with the analyticity structure of the amplitudes and make a statistical comparison to find whether the most favoured explanation of the anomalies is new physics or underestimated hadronic effects. Furthermore, assuming the source of the anomalies to be new physics, to get a fair view of how significantly favoured it is, we perform a global fit to all the Wilson coefficients which can effectively receive beyond the Standard Model contributions.

The International Conference on B-Physics at Frontier Machines - BEAUTY2018

6-11 May, 2018

La Biodola, Elba Island, Italy

\footnotetext{
* Speaker.
} 


\section{Introduction}

In recent years, several tensions have been observed between the Standard Model (SM) prediction of $b \rightarrow s \ell^{+} \ell^{-}$processes and the corresponding experimental measurements; tensions with more than $3 \sigma$ significance have been measured by the LHCb in the angular observable $P_{5}^{\prime}$ of the $B \rightarrow K^{*} \mu^{+} \mu^{-}$decay [1,2] and also in the branching ratio of the $B_{s} \rightarrow \phi \mu^{+} \mu^{-}$decay [3]. Other tensions with a significance of $2.2-2.6 \sigma$ have also been measured in the ratios $R_{K}$ and $R_{K^{*}}$ by the LHCb $[4,5]$, which if confirmed would establish the breaking of lepton flavour universality. Since the various observables are interdependent through the Wilson coefficients the preferred scenario which best explains the $b \rightarrow s \ell^{+} \ell^{-}$processes can be found out by performing global fits to all the relevant $b \rightarrow s$ data. Interestingly all the tensions can be explained with a common New Physics (NP) effect, as shown in global fits perfomed by several groups (see e.g. refs. [6-12]).

The $P_{5}^{\prime}$ observable was also measured by the CMS [18], ATLAS [19] and Belle [20] experiments, where the tension was confirmed by the latter two collaborations although with less significance which is mostly due to the larger experimental uncertainties compared to the LHCb results. The confirmation of the $P_{5}^{\prime}$ anomaly by other experiments makes it unlikely that the tension is due to statistical fluctuations and hence either underestimated hadronic effects or NP contributions are the most likely explanations [13-15]. The significance of the tension in $P_{5}^{\prime}$ however, depends on the precise treatment of the hadronic contributions which is still not completely settled $[14,16,17]$. The $B \rightarrow K^{*} \ell^{+} \ell^{-}$observables receive contributions from long-distance hadronic parts which are difficult to estimate. While at low $q^{2}$ the leading order hadronic contributions have been calculated in the QCD factorisation (QCDf) framework [21,22], the subleading nonfactorisable contributions remain unknown and are usually "guesstimated". However, there have been methods suggested for the estimation of these corrections using light-cone sum rule (LCSR) techniques and employing dispersion relations [23] and the analyticity structure of the amplitudes [24].

Furthermore, instead of making assumptions on the sizes of the power corrections, a general ansatz can be assumed with a number of unknown parameters [25-28] which is then fitted to the relevant data on $B \rightarrow K^{*} \mu^{+} \mu^{-}$and $B \rightarrow K^{*} \gamma$. Considering Wilks' theorem it is then possible to make a statistical comparison of the hadronic parameters fit and the NP fit of the Wilson coefficients.

Moreover, since a priori there is no reason to assume that the $b \rightarrow s \ell^{+} \ell^{-}$anomalies are due to only one type of New Physics contribution we assume NP to (simultaneously) appear in several operators and perform multidimensional fits where all the relevant Wilson coefficients receive contributions which can give a more fair picture of how significantly the anomalies indicate the existence of beyond the SM contributions.

\section{Short-distance versus long-distance contributions in $B \rightarrow K^{*} \ell^{+} \ell^{-}$}

The $b \rightarrow s \ell^{+} \ell^{-}$transitions can be described via the effective Hamiltonian

$$
\mathscr{H}_{\mathrm{eff}}=-\frac{4 G_{F}}{\sqrt{2}} V_{t b} V_{t s}^{*}\left\{\sum_{i=1, \ldots, 6,8} C_{i} O_{i}+\sum_{i=7,9,10, Q_{1}, Q_{2}, T}\left(C_{i} O_{i}+C_{i}^{\prime} O_{i}^{\prime}\right)\right\}
$$


For the exclusive decay $B \rightarrow K^{*} \mu^{+} \mu^{-}$, the semileptonic part of the Hamiltonian (second term in the braces) which accounts for the dominant contribution, can be factorised into a leptonic and hadronic piece where the latter can be described by seven independent form factors $\tilde{S}, \tilde{V}_{\lambda}, \tilde{T}_{\lambda}$, with helicities $\lambda= \pm 1,0$. The hadronic part of the Hamiltonian (first term in the braces) has a subleading contribution to $B \rightarrow K^{*} \mu^{+} \mu^{-}$via a virtual photon which decays into a lepton pair. This part of the Hamiltonian contains non-factorisable contributions and has a similar effect as the electromagnetic and the vectorial operators $\left(O_{7}\right.$ and $\left.O_{9}\right)$ and appears in the vectorial helicity amplitudes

$$
H_{V}(\lambda)=-i N^{\prime}\left\{C_{9}^{\mathrm{eff}} \tilde{V}_{\lambda}-C_{9}^{\prime} \tilde{V}_{-\lambda}+\frac{m_{B}^{2}}{q^{2}}\left[\frac{2 \hat{m}_{b}}{m_{B}}\left(C_{7}^{\mathrm{eff}} \tilde{T}_{\lambda}-C_{7}^{\prime} \tilde{T}_{-\lambda}\right)-16 \pi^{2} \mathscr{N}_{\lambda}\right]\right\}
$$

where the factorisable piece is described as the effective part of $C_{9}^{\text {eff }}\left(\equiv C_{9}+Y\left(q^{2}\right)\right)$ and the nonfactorisable piece is encoded by $\mathscr{N}_{\lambda}\left(q^{2}\right) \equiv$ Leading order in QCDf $+h_{\lambda}\left(q^{2}\right)$, with $h_{\lambda}$ denoting the unknown power corrections.

Since the short-distance NP contributions due to $\delta C_{9}^{\mathrm{NP}}$ (and/or $\delta C_{7}^{\mathrm{NP}}$ ) can be mimicked by long-distance effects in $h_{\lambda}$, a proper estimation of the size of the hadronic contributions is highly desirable and crucial in determining whether the observed anomalies in $B \rightarrow K^{*} \mu^{+} \mu^{-}$observables results in a significant NP interpretation.

\subsection{Theoretical approaches for calculation of the hadronic contributions}

There are different approaches suggested in the literature to estimate the hadronic contributions. In the "standard" method the hadronic contributions are estimated using the QCDf formalism where an expansion of $\Lambda / m_{b}$ is employed where the leading order factorisable and non-factorisable contributions are taken into account. However, higher powers of $\mathscr{O}\left(\Lambda / m_{b}\right)$ within the QCDf formalism are not known. In the so-called "full form factor" method (see i.e. Ref. [16]), only the power corrections to the non-factorisable piece in the QCDf formula remain unknown and are usually guesstimated to be $10 \%, 20 \%$ or even higher percentages compared to the leading nonfactorisable contributions.

The power correction, soft gluon effect, of the dominant hadronic contributions from the current-current operators $O_{1,2}$ have been estimated in Ref. [23] using the LCSR formalism in the $q^{2} \lesssim 1 \mathrm{GeV}^{2}$ region. The results are extrapolated up to the $J / \psi$ resonance by employing dispersion relations and using the experimental data from $B \rightarrow J / \psi K^{*}$ and $B \rightarrow \psi(2 S) K^{*}$ decays. However, in the theoretical input of the dispersion relation, the leading order non-factorisable effects which have an important contribution to the analyticity structure are not included (since part of the required calculations are not available in a flavour separated way). Moreover, the phases of the resonant amplitude relative to the short-distance contribution for each of the three amplitude structures (for both resonances) are assumed to be zero.

One way to compensate the missing leading order factorisable corrections in the Khodjamirian et al. method is to just add these missing contributions to the phenomenological model. This is done for example in Ref. [11] (referred to as PMD). However, the theoretical error which enters this procedure is rather unclear.

In Ref. [24], the authors consider the analyticity of the amplitude and building upon the work of refs. [23,29], both the leading and subleading hadronic contributions arising from the $O_{1,2}$ opera- 
tors have been estimated. The calculations are performed at $q^{2}<0$ where the theory predictions for the leading terms in QCDf $[21,22,30,31]$ as well as the subleading terms in LCSR [23,29] are reliable and in combination with the experimental information on the $B \rightarrow J / \psi K^{*}$ and $B \rightarrow \psi(2 S) K^{*}$ decays, the hadronic contributions from the charm loops are estimated in the physical region up to the $\psi(2 S)$ resonance.

\subsection{Comparison of the different approaches}

To examine how the various theory estimations differ in their predictions of $B \rightarrow K^{*} \mu^{+} \mu^{-}$ observables, the SM predictions for $d \mathrm{BR} / d q^{2}$ and $P_{5}^{\prime}$ using the various implementations of the hadronic contributions are given in Fig. 1. In the "standard" method, the predictions are given for below $q^{2}=8 \mathrm{GeV}^{2}$ where QCDf calculations are reliable while the phenomenological model of Khodjamirian et al. is considered up to $q^{2}<9 \mathrm{GeV}^{2}$ and only the Bobeth et al. method has a prediction for also between the $J / \psi$ and $\psi(2 S)$ resonances. Interestingly the central values of the latter two methods increase the tension with the experimental results for both $d \mathrm{BR} / d q^{2}$ and $P_{5}^{\prime}$ and it seems that the contributions from the power corrections tend to further escalate the tension with the data. The theoretical errors of these predictions however, are larger (for the Bobeth et al. method the large theoretical uncertainty is due to not including the correlations among uncertainties as they are not provided in Ref. [24]).
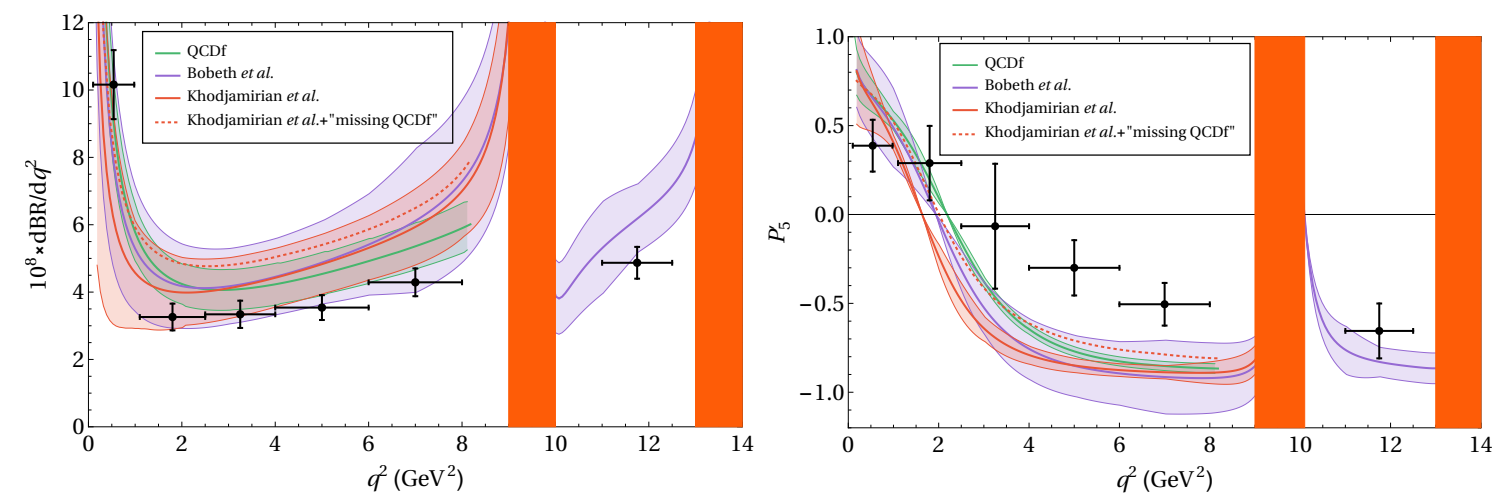

Figure 1: The SM predictions of $d \mathrm{BR} / q^{2}$ and $P_{5}^{\prime}$ observables of the $B \rightarrow K^{*} \mu^{+} \mu^{-}$decays within various implementations of the hadronic contributions. For the "QCDf" implementation the full form factor method has been considered, with a $10 \%$ error assumption for the power corrections. The theory error of the Khodjamirian et al. and also the Bobeth et al. implementations are obtained by considering the relevant parameter uncertainties. The theoretical uncertainty of the method where the leading order non-factorisable contributions are added to the phenomenological model of Ref. [23] (Khodjamirian et al. + "missing QCDf") are not shown.

In Table 1 the significance of different NP scenarios (for one operator fits to $\delta C_{7}, \delta C_{9}$ or $\delta C_{10}$ ) are given using the "standard" implementation (with $10 \%$ error assumption on the power corrections) and the Bobeth et al. implementation of the non-factorisable corrections. While in both implementations New Physics contribution to $C_{9}$ constitutes the favoured scenario, the significance and the best fit values are different. 


\begin{tabular}{l|c||c|c||c|c||c|c}
\multicolumn{1}{|c|}{} & \multicolumn{2}{|c||}{$\mathrm{SM}$} & \multicolumn{2}{c||}{$\delta C_{7}$} & \multicolumn{2}{c}{$\delta C_{9}$} & \multicolumn{2}{c}{$\delta C_{10}$} \\
\cline { 2 - 7 } & $\chi_{\min }^{2}$ & b.f. value & $\chi_{\min }^{2}$ & b.f. value & $\chi_{\min }^{2}$ & b.f. value & $\chi_{\min }^{2}$ \\
\hline QCDf & 60.9 & $-0.03 \pm 0.02$ & $58.9(1.4 \sigma)$ & $-1.05 \pm 0.21$ & $45.4(3.9 \sigma)$ & $-0.17 \pm 0.35$ & $60.7(0.5 \sigma)$ \\
Bobeth et al. & 54.8 & $-0.03 \pm 0.03$ & $53.5(1.1 \sigma)$ & $-1.26 \pm 0.28$ & $43.9(3.3 \sigma)$ & $0.48 \pm 0.63$ & $54.1(0.8 \sigma)$
\end{tabular}

Table 1: $\chi^{2}$ of the one operator NP fit compared to the SM within the "standard" QCDf method (with a $10 \%$ error assumption on the power corrections) and the Bobeth et al. method. The observables considered in the fit include $B R\left(B \rightarrow K^{*} \gamma\right), B R\left(B^{+} \rightarrow K^{+*} \mu^{+} \mu^{-}\right)$in the [1.1-6.0] and [15-19] GeV bins and all $B \rightarrow K^{*} \mu^{+} \mu^{-}$observables in both low and high $q^{2}$ bins.

\section{General ansatz for the power corrections}

Instead of employing the (yet unsettled) theoretical methods for estimating the power corrections or making ad hoc assumptions on their sizes, they can be parameterised by a polynomial with a number of free parameters which can be fitted to the experimental data [25]. In our previous work [26], we assumed a general $q^{2}$-polynomial ansatz

$$
h_{\lambda}\left(q^{2}\right)=h_{\lambda}^{(0)}+\frac{q^{2}}{1 \mathrm{GeV}^{2}} h_{\lambda}^{(1)}+\frac{q^{4}}{1 \mathrm{GeV}^{4}} h_{\lambda}^{(2)} .
$$

where we used the measurements on $B \rightarrow K^{*} \mu^{+} \mu^{-}$observables to determine the free parameters. Since we now also consider the experimental result on $\operatorname{BR}\left(B \rightarrow K^{*} \gamma\right)$, compatibility with the analytical structure for $q^{2} \rightarrow 0$ is mandatory. Hence, while keeping the same ansatz for $\lambda= \pm$ we have modified the $h_{\lambda}\left(q^{2}\right)$ ansatz for $\lambda=0$ (to avoid producing a physical pole in the longitudinal amplitude at $q^{2}=0$ )

$$
h_{0}\left(q^{2}\right)=\sqrt{q^{2}} \times\left(h_{0}^{(0)}+\frac{q^{2}}{1 \mathrm{GeV}^{2}} h_{0}^{(1)}+\frac{q^{4}}{1 \mathrm{GeV}^{4}} h_{0}^{(2)}\right) .
$$

This modified definition for $h_{\lambda}$ is the most general ansatz for the unknown hadronic contributions (up to higher order powers in $q^{2}$ ) which is compatible with the analyticity structure assumed in Ref. [24] and can also be used to paramterise the unknown hadronic contributions in the radiative $B \rightarrow K^{*} \gamma$ decay.

\subsection{Hadronic fit vs NP fit to $\delta C_{7,9}$}

In order to investigate whether the $B \rightarrow K^{*} \mu^{+} \mu^{-}$data are better explained by assuming NP or underestimated hadronic contributions, we have done separate fits for each case where only the low $q^{2}$ data have been used. For the hadronic fit, we have varied the 18 free parameters describing the complex $h_{+,-, 0}^{(0,1,2)}$. Most of the fitted parameters are consistent with zero as they have large uncertainties (see Table 1 in Ref. [32]), however, this can be changed with more precise experimental results and finer $q^{2}$ binning. Using the same set of observables we also made one and two operator NP fit to $\delta C_{9}$ and $\delta C_{7,9}$ assuming the Wilson coefficients to be either real or complex (see Table 2 in Ref. [32]). In all four NP scenarios there is a larger than $4 \sigma$ significance better description of the data compared to the SM hypothesis. 
As was explicitly shown in Ref. [32], the effect of New Physics contributions due to $C_{7}$ and $C_{9}$ can be embedded in the more general case of the hadronic contributions. Due to the embedding, there can be a statistical comparison between the NP fit versus the hadronic contribution fit. In Table 2 the significance of the improvement of the fit in the hypothesis with more free parameters has been compared to that with less free parameters using the Wilks' theorem. While the hadronic solution and the NP explanation both have a better description of the measured data with a significance of larger than $3 \sigma$, there is always less than $1.5 \sigma$ improvement when going from the NP fit to the hadronic one.

\begin{tabular}{l|c|c|c|c|c}
$\begin{array}{l}\text { nr. of free } \\
\text { parameters }\end{array}$ & 1 & 2 & 2 & 4 & 18 \\
\hline 0 (plain SM) & $4.1 \sigma$ & $4.0 \sigma$ & $4.2 \sigma$ & $4.1 \sigma$ & $3.1 \sigma$ \\
$1\left(\right.$ Real $\left.\delta C_{9}\right)$ & - & $1.5 \sigma$ & $2.1 \sigma$ & $2.0 \sigma$ & $1.5 \sigma$ \\
$2\left(\right.$ Real $\left.\delta C_{7}, \delta C_{9}\right)$ & - & - & - & $1.9 \sigma$ & $1.4 \sigma$ \\
$2\left(\right.$ Complex $\left.\delta C_{9}\right)$ & - & - & - & $1.4 \sigma$ & $1.1 \sigma$ \\
$4\left(\right.$ Complex $\left.\delta C_{7}, \delta C_{9}\right)$ & - & - & - & - & $0.95 \sigma$
\end{tabular}

Table 2: Improvement of the hadronic fit and the scenarios with real and complex NP contributions to $C_{7}$ and $C_{9}$ compared to the SM hypothesis and compared to each other. For the fits $B R\left(B \rightarrow K^{*} \gamma\right), \operatorname{BR}\left(B^{+} \rightarrow\right.$ $\left.K^{+*} \mu^{+} \mu^{-}\right)_{q^{2} \in[1.1-6.0] \mathrm{GeV}^{2}}$ and the $C P$ averaged observables of the $B \rightarrow K^{*} \mu^{+} \mu^{-}$decays in the low $q^{2}$ bins up to $8 \mathrm{GeV}^{2}$ have been considered.

Hence it can be concluded that the hadronic fit which has an additional 14-17 more parameters compared to the New Physics fit does not significantly improve the fit. Thus, at the moment the statistical comparison favours the NP explanation. However, the situation stays inconclusive since with the set of observables considered in this analysis, the NP fit can be embedded in the hadronic fit; in this case one cannot disprove the hadronic option in favour of the NP one ${ }^{1}$. Moreover, with the present results, there is no indication that higher powers of $q^{2}$ than what is attainable by NP contributions to $C_{7}$ and $C_{9}$ would be required to explain the $B \rightarrow K^{*} \mu^{+} \mu^{-}$data. However, this might be due to the size of the current $q^{2}$ bins which can potentially smear out a significant $q^{2}$ dependence and thus smaller binning can shed more light on this issue.

Assuming NP to be responsible for the observed deviations, several groups have performed global fits to $b \rightarrow s$ data, with slight differences in their SM predictions which arise from the different employed theory approaches as well as from the difference in the input parameters (e.g. which sets of form factor calculations are used). Moreover, in the various global fits, slightly different sets of observables are considered. Nonetheless, as long as the non-factorsible contributions are not assumed to be very large, the overall global fit results indicate a consistent picture where NP in $C_{9}^{\mu}$ is the favoured scenario (see e.g. Refs. [6-12]).

\footnotetext{
${ }^{1}$ More precise measurements on CP-asymmetric $B \rightarrow K^{*} \mu^{+} \mu^{-}$observables can break the embedding as the imaginary parts in Wilson coefficients correspond to CP-violating "weak" phases while in the hadronic contributions correspond to CP-conserving "strong" phases [33].
} 


\section{Fit to NP including scalar \& pseudoscalar operators}

Presuming that the observed tensions in the $b \rightarrow s \ell^{+} \ell^{-}$transitions are due to New Physics contributions there is in principle no reason that these effects should only contribute via one or two Wilson coefficients. In particular, a complete NP scenario incorporates many new particles and can have extended Higgs sectors, affecting the Wilson coefficients $C_{7 \cdots 10}$ and requiring scalar and pseudoscalar contributions.

We have thus expanded our study to include NP in the global fit to all $b \rightarrow s \ell^{+} \ell^{-}$data (see Ref. [32] for the considered observables) from all the relevant Wilson coefficients $C_{7}, C_{8}, C_{9}^{\ell}, C_{10}^{\ell}$, $C_{Q_{1}}^{\ell}, C_{Q_{2}}^{\ell}$ (assuming lepton flavours to be $\ell=e, \mu$ ) for the first time. When considering the chiralityflipped counterparts of the operators, this results in 20 Wilson coefficients in the fit. To perform our fits, the theoretical correlations and errors are computed using SuperIso v 4.0 [34,35], which incorporates an automatic multiprocessing calculation of the covariance matrix for each parameter point. We have considered $10 \%$ error assumption for the power corrections. It is often considered that the data on $\operatorname{BR}\left(B_{s} \rightarrow \mu^{+} \mu^{-}\right)$remove the possibility to have large scalar and pseudoscalar Wilson coefficients $C_{Q_{1,2}}$. While this is rather true for $C_{Q_{1}}$, there exists a degeneracy between $C_{10}$ and $C_{Q_{2}}$ which makes it possible to have simultaneously large values for both Wilson coefficients.

We first consider NP contribution only in $C_{9}^{\mu}$ and then expand the fit, varying simultaneously 2, 6,10 and 20 Wilson coefficients. The results of the fits are given in Table. 3 where the last column corresponds to the improvement in comparison to the previous set of Wilson coefficients, obtained using the Wilks' theorem. The best fit values of the one- and the multi-dimensional fits can be found in Ref. [32]. The pull with the SM increases with the number of Wilson coefficients which is due to the increase in the number of degrees of freedom. However there is no real improvement in the fit when adding more Wilson coefficients compared to the " $C_{9}^{\mu}$ only" set. As a result of the full fit to all available $b \rightarrow s$ data including all the relevant Wilson coefficients, a total pull of $4.1 \sigma$ with the SM hypothesis is obtained, assuming $10 \%$ error for the power corrections.

\begin{tabular}{c|c|c|c|c} 
Set of WC & Nr. parameters & $\chi_{\min }^{2}$ & Pull & Improvement \\
\hline SM & 0 & 118.8 & - & - \\
$C_{9}^{\mu}$ & 1 & 85.1 & $5.8 \sigma$ & $5.8 \sigma$ \\
$C_{9}^{(e, \mu)}$ & 2 & 83.9 & $5.6 \sigma$ & $1.1 \sigma$ \\
$C_{7}, C_{8}, C_{9}^{(e, \mu)}, C_{10}^{(e, \mu)}$ & 6 & 81.2 & $4.8 \sigma$ & $0.5 \sigma$ \\
All non-primed WC & $10(8)$ & 81.0 & $4.1(4.5) \sigma$ & $0.0(0.1) \sigma$ \\
All WC (incl. primed) & $20(16)$ & 70.2 & $3.6(4.1) \sigma$ & $0.9(1.2) \sigma$
\end{tabular}

Table 3: The $\chi_{\min }^{2}$ values when varying different Wilson coefficients. In the last column the significance of the improvement of the fit compared to the scenario of the previous line is given. The numbers in the parenthesis correspond to removing $C_{Q_{1,2}}^{e \prime}$ from the relevant fits.

\section{Future prospects}

The LHCb detector will be upgraded and is expected to collect a total integrated luminosity of 


\begin{tabular}{l|c|c|c}
$\Delta C_{9}^{\mu}$ & $\begin{array}{c}\text { Pull } \\
\text { SM }\end{array}$ & $\begin{array}{c}\text { Pull }_{\mathrm{SM}} \\
\text { Syst. }\end{array}$ & $\begin{array}{c}\text { Pull } \\
\text { SM }\end{array}$ \\
\hline $12 \mathrm{fb}^{-1}$ & $6.1 \sigma(4.3 \sigma)$ & $7.2 \sigma(5.2 \sigma)$ & $7.4 \sigma(5.5 \sigma)$ \\
$50 \mathrm{fb}^{-1}$ & $8.2 \sigma(5.7 \sigma)$ & $11.6 \sigma(8.7 \sigma)$ & $12.9 \sigma(9.9 \sigma)$ \\
$300 \mathrm{fb}^{-1}$ & $9.4 \sigma(6.5 \sigma)$ & $15.6 \sigma(12.3 \sigma)$ & $19.5 \sigma(16.1 \sigma)$
\end{tabular}

Table 4: Pull $\mathrm{SM}_{\mathrm{S}}$ for the fit to $\Delta C_{9}^{\mu}$ based on the ratios $R_{K}$ and $R_{K^{*}}$ for the LHCb upgrade scenarios assuming current central values. The systematic error is considered to either remain unchanged or be reduced by $a$ factor of 2 or 3 . The three $R_{K}$ and $R_{K^{*}}$ bins/observables are assumed to have no correlation (50\% correlation between each of the three measurements).

$50 \mathrm{fb}^{-1}$. A second upgrade at a high-luminosity LHC will allow for a full dataset of up to $300 \mathrm{fb}^{-1}$, leading to a decrease of the statistical errors by a factor of 4 and 10 respectively. We consider three scenarios in which the current central values are assumed to remain and in which the systematic error is either unchanged or reduced by a factor of 2 or 3 . In all cases we consider two (extreme) options regarding the error correlations, namely that the three $R_{K}$ and $R_{K^{*}}$ bins/observables have no correlation or $50 \%$ correlation between each of the three measurements. The results for these future scenarios are given in Table 4. Here we show the one-operator NP hypothesis $\Delta C_{9}^{\mu}$ as an exemplary mode. It is obvious from the SM pulls that - within the scenario in which the central values are assumed to remain - only a small part of the $50 \mathrm{fb}^{-1}$ is needed to establish NP in the $R_{K^{(*)}}$ ratios even in the pessimistic case that the systematic errors are not reduced by then at all.

We also consider the set of $b \rightarrow s \ell \ell$ observables, which is complementary to $R_{K}$ and $R_{K}^{*}$, again assuming the central values remain. Future prospects are given for two operator fits in Fig. 2. Under this assumption it seems possible that the LHCb collaboration will be able to establish new physics within the angular observables even in the pessimistic case that there will be no theoretical progress on non-factorisable power corrections.

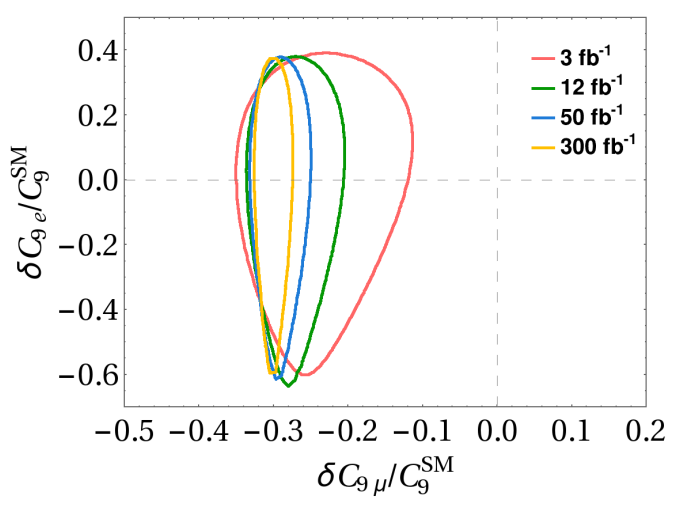

Figure 2: Global fit results for $\delta C_{9}^{e}-\delta C_{9}^{\mu}$ using all $b \rightarrow s \bar{\ell} \ell$ observables (under the assumption of $10 \%$ factorisable power corrections) besides $R_{K}$ and $R_{K^{*}}$. Future LHCb prospects of the fit (at $2 \sigma$ level), assuming the current central values remain, are shown for different luminosities. 


\section{Conclusions}

We explored the available state-of-the-art methods for implementing the power corrections and demonstrated that while the various methods offer different SM predictions and uncertainties, in all these cases, in the critical bin where the $P_{5}^{\prime}$ anomaly is observed, the predictions roughly converge giving prominence to the observed tension.

We also assumed the most general parameterisation (up to higher $q^{2}$ terms) for the power corrections (modeled with 18 free parameters) and fitted the free parameters to the experimental data and showed that in comparison with the NP fit to (real or complex) $C_{9}$ and $C_{7}$ Wilson coefficients (with 1-4 free parameters) there is no significant preference in adding 14-17 parameters which indicated that NP is the favoured explanation of the anomalies. This is however, partly due to the experimental results not being constraining enough so that most of the hadronic parameters are consistent with zero and also since possible preference for a large $q^{2}$-dependence might be masked due to the $q^{2}$ smearing within the current ranges of the bins.

Furthermore, we presented global fits to all the $b \rightarrow s$ data where all the relevant Wilson coefficients can simultaneously receive NP contributions. The various 1, 2, 6, 10 and 20 dimensional fits when varying different Wilson coefficients do not indicate any preference for NP beyond $C_{9}$. At the moment, a large number of Wilson coefficients are very loosely bound or completely undetermined in the case of electron scalar and pseudoscalar operators. This is interesting since especially with the indication of lepton flavour universality violation from the $R_{K}$ and $R_{K^{*}}$ ratios, there is motivation to investigate the electron and muon sectors separately for the scalar and pseudoscalar operators.

Finally, we discussed the LHCb future prospects for establishing the source of the observed anomalies. The future measurements of the theoretically clean ratios $R_{K^{(*)}}$ and similar observables which are sensitive to lepton flavour non-universality have the potential to unambiguously establish lepton non-universal new physics in the near future.

\section{Acknowledgements}

F.M. would like to thank the organisers of BEAUTY2018 for their invitation and for the very fruitful conference.

\section{References}

[1] R. Aaij et al. [LHCb Collaboration], Phys. Rev. Lett. 111, 191801 (2013)

[2] R. Aaij et al. [LHCb Collaboration], JHEP 1602, 104 (2016)

[3] R. Aaij et al. [LHCb Collaboration], JHEP 1509, 179 (2015)

[4] R. Aaij et al. [LHCb Collaboration], Phys. Rev. Lett. 113, 151601 (2014)

[5] R. Aaij et al. [LHCb Collaboration], JHEP 1708, 055 (2017)

[6] B. Capdevila, A. Crivellin, S. Descotes-Genon, J. Matias and J. Virto, JHEP 1801, 093 (2018)

[7] W. Altmannshofer, P. Stangl and D. M. Straub, Phys. Rev. D 96, no. 5, 055008 (2017) 
[8] G. D’Amico, M. Nardecchia, P. Panci, F. Sannino, A. Strumia, R. Torre and A. Urbano, JHEP 1709, 010 (2017)

[9] G. Hiller and I. Nisandzic, Phys. Rev. D 96, no. 3, 035003 (2017)

[10] L. S. Geng, B. Grinstein, S. Jäger, J. Martin Camalich, X. L. Ren and R. X. Shi, Phys. Rev. D 96, no. 9, 093006 (2017)

[11] M. Ciuchini, A. M. Coutinho, M. Fedele, E. Franco, A. Paul, L. Silvestrini and M. Valli, Eur. Phys. J. C 77, no. 10, 688 (2017)

[12] T. Hurth, F. Mahmoudi, D. Martinez Santos and S. Neshatpour, Phys. Rev. D 96, no. 9, 095034 (2017)

[13] S. Jäger and J. Martin Camalich, JHEP 1305, 043 (2013)

[14] T. Hurth and F. Mahmoudi, JHEP 1404, 097 (2014)

[15] F. Mahmoudi, S. Neshatpour and J. Virto, Eur. Phys. J. C 74, no. 6, 2927 (2014)

[16] T. Hurth, F. Mahmoudi and S. Neshatpour, Nucl. Phys. B 909, 737 (2016)

[17] F. Mahmoudi, T. Hurth and S. Neshatpour, Nucl. Part. Phys. Proc. 285-286, 39 (2017)

[18] CMS Collaboration, A. M. Sirunyan et al., Phys. Lett. B781 (2018) 517-541,

[19] ATLAS Collaboration, M. Aaboud et al., arXiv:1805.04000 [hep-ex] .

[20] Belle Collaboration, A. Abdesselam et al., Proceedings, LHCSki 2016 - A First Discussion of 13 TeV Results: Obergurgl, Austria, April 10-15, 2016. arXiv:1604.04042 [hep-ex].

[21] M. Beneke, T. Feldmann and D. Seidel, Nucl. Phys. B 612, 25 (2001)

[22] M. Beneke, T. Feldmann and D. Seidel, Eur. Phys. J. C 41, 173 (2005)

[23] A. Khodjamirian, T. Mannel, A. A. Pivovarov and Y.-M. Wang, JHEP 1009, 089 (2010)

[24] C. Bobeth, M. Chrzaszcz, D. van Dyk and J. Virto, Eur. Phys. J. C 78, 6 (2018)

[25] M. Ciuchini, M. Fedele, E. Franco, S. Mishima, A. Paul, L. Silvestrini and M. Valli, JHEP 1606, 116 (2016)

[26] V. G. Chobanova, T. Hurth, F. Mahmoudi, D. Martinez Santos and S. Neshatpour, JHEP 1707, 025 (2017)

[27] S. Neshatpour, V. G. Chobanova, T. Hurth, F. Mahmoudi and D. Martinez Santos, arXiv:1705.10730 [hep-ph].

[28] F. Mahmoudi, T. Hurth and S. Neshatpour, Acta Phys. Polon. B 49, 1267 (2018).

[29] A. Khodjamirian, T. Mannel and Y. M. Wang, JHEP 1302, 010 (2013)

[30] H. H. Asatrian, H. M. Asatrian, C. Greub and M. Walker, Phys. Lett. B 507, 162 (2001)

[31] H. H. Asatryan, H. M. Asatrian, C. Greub and M. Walker, Phys. Rev. D 65, 074004 (2002)

[32] A. Arbey, T. Hurth, F. Mahmoudi and S. Neshatpour, arXiv:1806.02791 [hep-ph].

[33] C. Bobeth, G. Hiller and G. Piranishvili, JHEP 0807, 106 (2008)

[34] F. Mahmoudi, Comput. Phys. Commun. 178 (2008) 745

[35] F. Mahmoudi, Comput. Phys. Commun. 180 (2009) 1579 